\title{
Noninvasive 24 hour ambulatory blood pressure monitoring: current status
}

\author{
Alice Stanton and Eoin O'Brien
}

\author{
Blood Pressure Unit, Beaumont Hospital, Dublin 9, Ireland
}

\section{Introduction}

Noninvasive intermittent blood pressure monitoring was first developed 30 years ago. ${ }^{1}$ It is now evolving from its former role as a somewhat esoteric research tool to becoming a clinically useful procedure for the evaluation of hypertensive patients. In this review we outline its historical development, compare noninvasive 24-hour ambulatory blood pressure monitoring with the other available methods of blood pressure assessment, and evaluate the current roles for the technique in the diagnosis and management of hypertension.

\section{Historical backround}

Arterial blood pressure may be measured directly by intra-arterial puncture, or indirectly by noninvasive means, by manual or automated techniques, and in stationary or ambulant subjects. The Reverend Steven Hales was the first to measure blood pressure directly in animals. He showed that blood rose to a height of over 8 feet in a glass tube placed in the artery of a horse. ${ }^{2} \mathrm{~A}$ device suitable for measuring human intra-arterial pressure was developed by Jean-Leonard Marie Poiseuille in $1828,{ }^{3}$ but it was not until 1969 that the first report of automated intra-arterial blood pressure recording in unrestricted ambulant man was published. ${ }^{4}$ The essential components for continuous intraarterial pressure measurement using the popular 'Oxford System's are a fine intra-arterial catheter (in the brachial or radial artery) constantly perfused, a miniaturized pressure tranducer and a magnetic tape recorder. This system can provide a complete accurate profile of blood pressure behaviour, measuring each individual beat over 24 hours, delineating beat-to-beat variability and circadian rhythms clearly in subjects performing

Correspondence: Professor E. O'Brien, M.D., F.R.C.P. (Lond.), F.R.C.P. (Ed.), F.A.C.C.P.

Received: 7 December 1992 normal daily activities. Due to its invasive nature, the impact of direct inter-arterial blood pressure recording on clinical practice has been limited. Attention has thus turned to noninvasive or indirect techniques to obtain a profile of 24-hour blood pressure.

The first instrument for measuring blood pressure indirectly was devised by Jules Herisson in 1833 , and consisted of a mercury reservoir covered by a rubber membrane from which a graduated glass column arose. The mercury bulb was compressed against the radial artery until the oscillations in the mercury column ceased, at which point systolic pressure was estimated. Many steps, too numerous to describe, have punctuated the development of modern indirect devices. ${ }^{6}$ Currently used devices use arm arterial occlusion ${ }^{7}$ and detect blood pressure level by Korotkov sound auscultation, ${ }^{8}$ oscillometry ${ }^{9}$ or ultrasonography. ${ }^{10}$ These indirect techniques of blood pressure measurement may be utilized in three ways - for conventional measurement of blood pressure in the clinic or surgery setting; for self-measurement of blood pressure usually in the home environment, and for automated measurement of blood pressure over the 24-hour period - each providing somewhat different information.

Clinical blood pressure measurements by doctors and nurses have been used since the turn of the century, but self-measurement of blood pressure by the patient has only been extensively explored in the last decade. ${ }^{11-14}$

In 1962 Hinman and his colleagues described the first truly portable system for the intermittent noninvasive measurement of blood pressure in subjects performing their usual daily routine. ${ }^{1}$ This system was developed commercially by the Remler company in California ${ }^{15}$ and consisted of a batteryoperated recorder worn by the patient, a cuff which was inflated by the patient at predetermined intervals and a microphone strapped over the brachial artery. Blood pressures were recorded on a magnetic tape, which could be later decoded, and the pressure plotted over the period of recording. 
Because the device depended on inflation by the subject, recordings were confined to waking hours and rarely spanned more than 12-14 hours. With the development of compact pumps and solid-state memory systems, the Remler system has been replaced by devices capable of automatically inflating the cuff and providing pressure profiles over 24 hours such as those illustrated in Figures 1-6. The early models were noisy and bulky, but latterly they have become smaller, quieter and more 'patient friendly'. Also the number of such devices has increased. There are now at least 15 systems currently available ${ }^{16}$ and many more in the developmental stages.

Noninvasive blood pressure monitoring systems suffer from the disadvantage that during measurement the subject must discontinue all activity and provide support for the arm, and hence the measurements are static rather than truely 'ambulatory'. Additionally only an intermittent, as opposed to a continuous record of 24-hour blood pressure behaviour is provided. Dispite these limitations noninvasive monitoring systems yield mean 24-hour values and diurnal/nocturnal patterns which are surprisingly close to those measured by continuous intra-arterial monitoring. ${ }^{17}$

Some attempts have been made to develop systems that provide indirect continuous 24 -hour blood pressure monitoring during rest and activity $^{18,19}$ - in short the equivalent of direct intra-arterial measurement without the inherent dangers of arterial catheterization - but no such system has as yet proved sufficiently accurate. Hence the remainder of this review will focus on the status of noninvasive intermittent 24-hour 'ambulatory' blood pressure monitoring.

\section{Device validation}

The instruments for noninvasive pressure monitoring are expensive, both in terms of capital and running costs. As a result, it has become increasingly important that devices are shown to be accurate. In order to minimize reliance on manufacturers' reports, two groups have published validation protocols, the Association for the Advancement of Medical Instrumention ${ }^{20}$ and the British Hypertension Society. ${ }^{21}$ The Association for the Advancement of Medical Instrumentation (AAMI) criteria stipulate that the test device should not differ from the mercury measurement by more than $5 \mathrm{mmHg}$, with a standard deviation of less than $8 \mathrm{mmHg}$. The British Hypertension Society (BHS) protocol grades each device according to the percentage of measurements differing from the mercury standard by 5,10 or $15 \mathrm{mmHg}$ oo

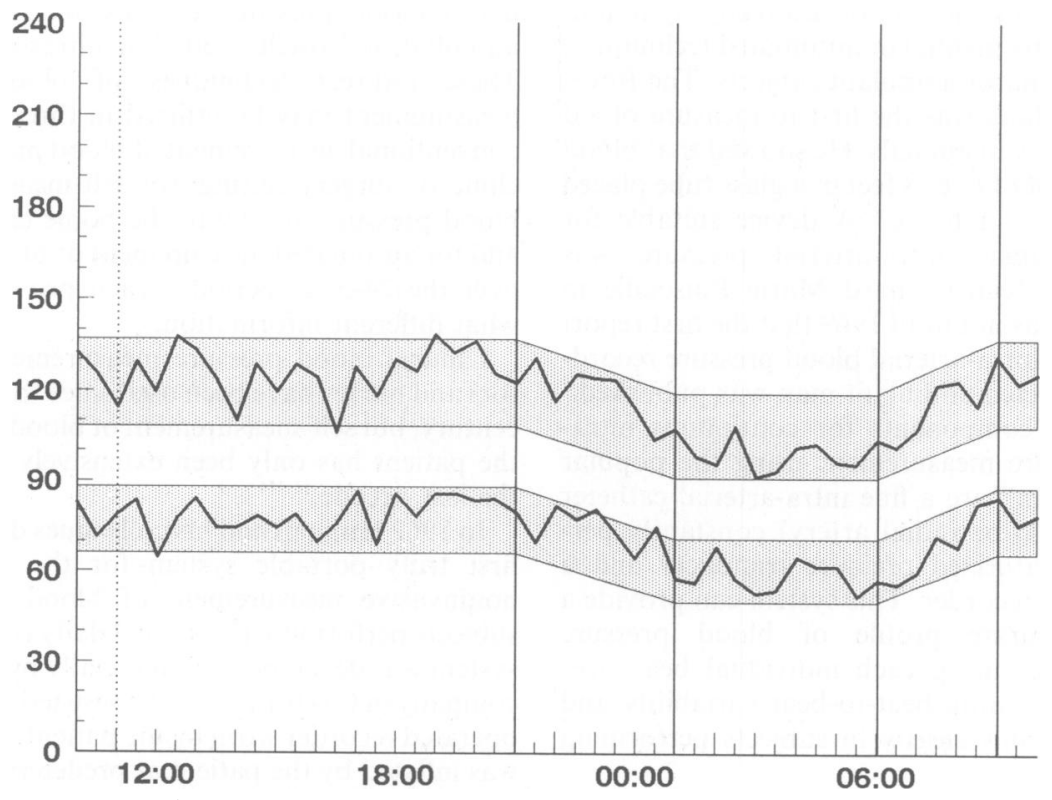

Figure 1 Twenty-four-hour ambulatory blood pressure profile from a normotensive subject with a normal circadian pattern. Time (hours) is plotted from the horizontal axis and blood pressure ( $\mathrm{mmHg}$ ), both systolic and diastolic, from the vertical axis. Normal values ${ }^{97}$ are indicated by the shaded bands and the vertical lines delineate initial, daytime, evening, night-time and early morning periods. 


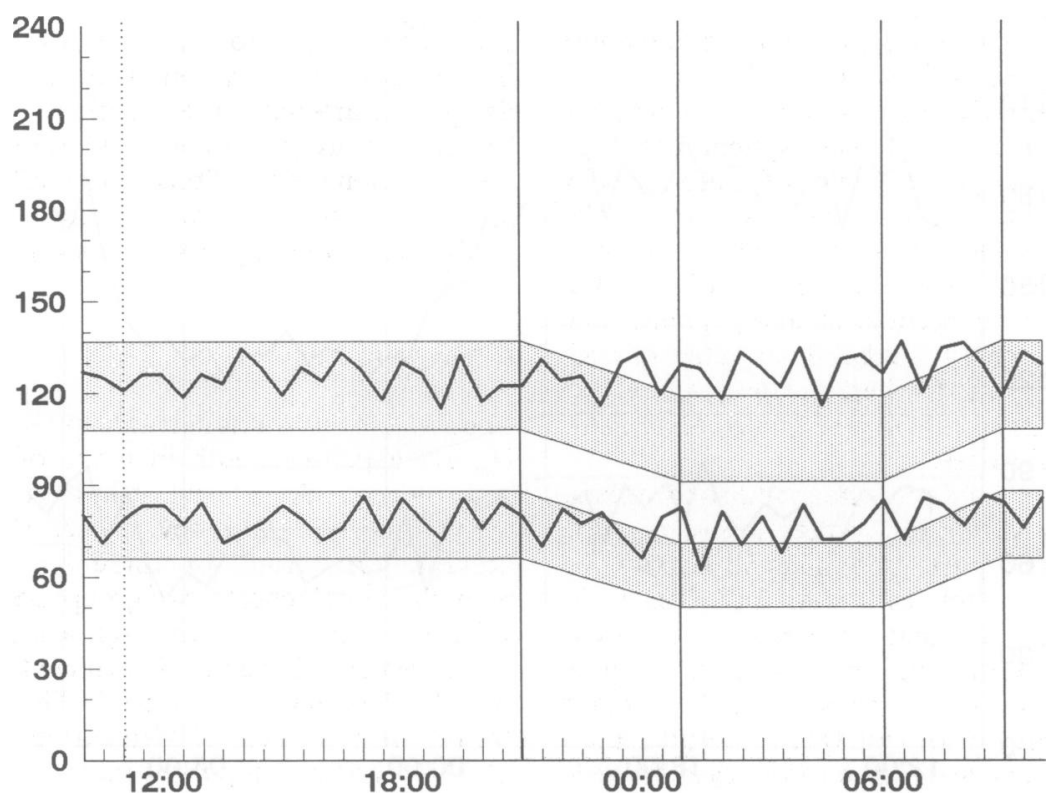

Figure 2 Twenty-four-hour ambulatory blood pressure profile from a subject with a blunted circadian pattern. Time (hours) is plotted from the horizontal axis and blood pressure ( $\mathrm{mmHg}$ ), both systolic and diastolic, from the vertical axis. Normal values ${ }^{97}$ are indicated by the shaded bands, and the vertical lines delineate initial, daytime, evening, night-time and early morning periods.

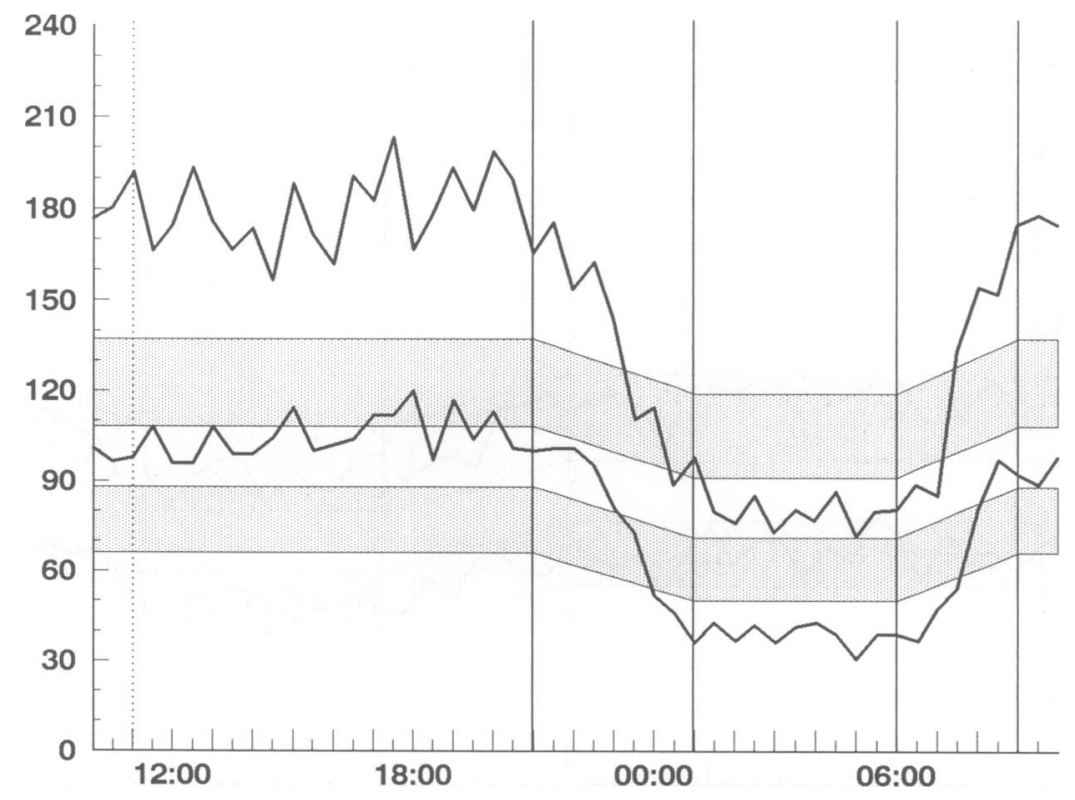

Figure 3 Twenty-four-hour ambulatory blood pressure profile from a subject with an accentuated circadian pattern. Time (hours) is plotted from the horizontal axis and blood pressure $(\mathrm{mmHg})$, both systolic and diastolic, from the vertical axis. Normal values ${ }^{97}$ are indicated by the shaded bands, and the vertical lines delineate initial, daytime, evening, night-time and early morning periods. 


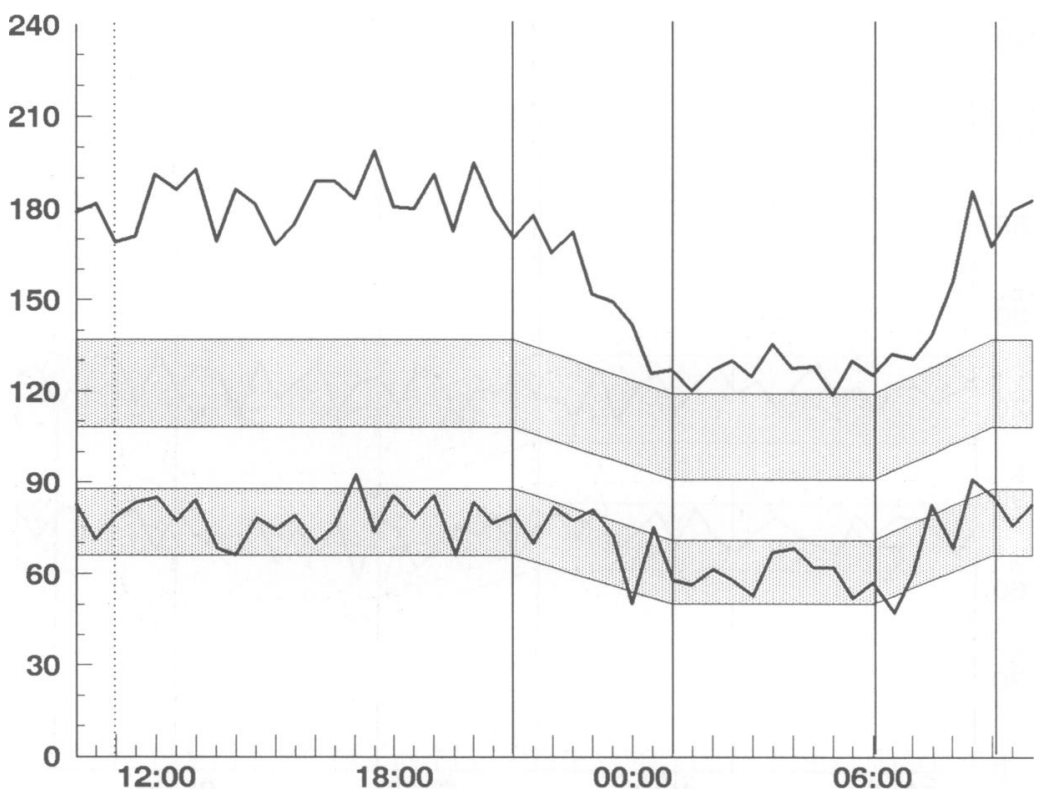

Figure 4 Twenty-four-hour ambulatory blood pressure profile from a subject with marked systolic hypertension but normal diastolic blood pressure. Time (hours) is plotted from the horizontal axis and blood pressure ( $\mathrm{mmHg}$ ), both systolic and diastolic, from the vertical axis. Normal values ${ }^{97}$ are indicated by the shaded bands, and the vertical lines delineate initial, daytime, evening, night-time and early morning periods.

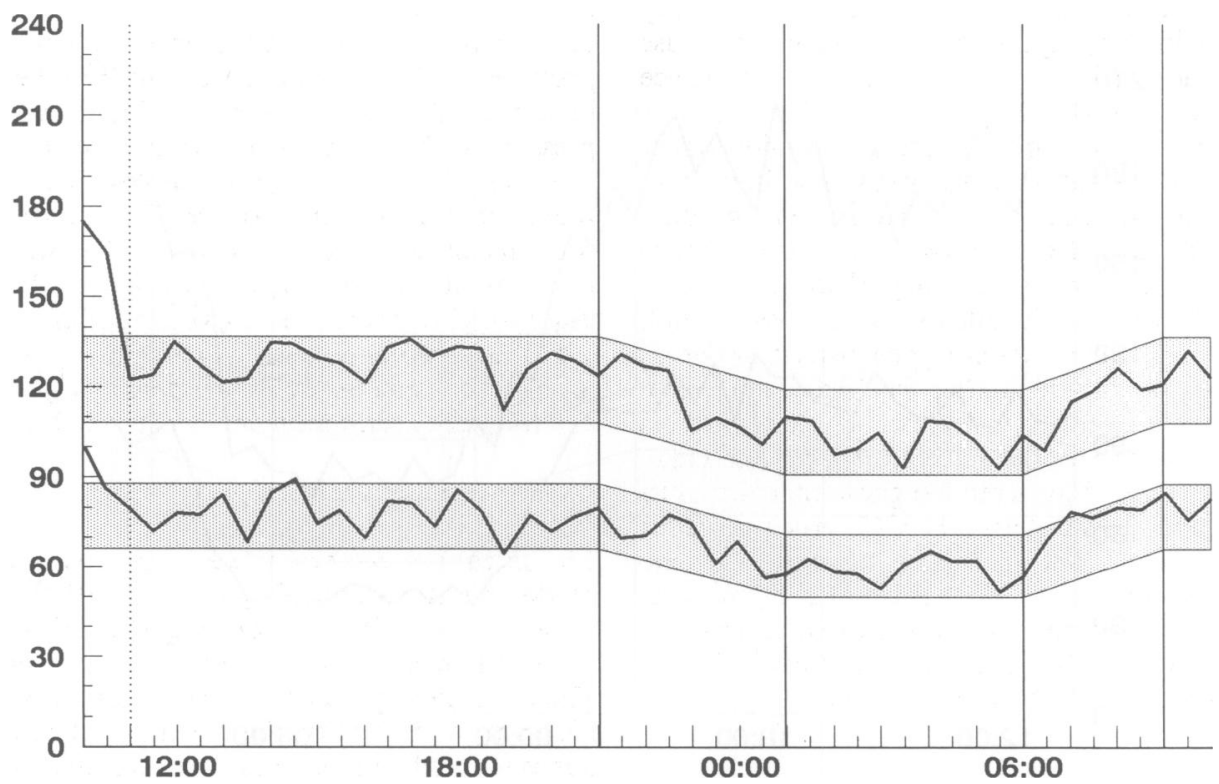

Figure 5 Twenty-four-hour ambulatory blood pressure profile from a subject with white-coat hypertension - only the initial blood pressure readings lie outside normotensive range. Time (hours) is plotted from the horizontal axis and blood pressure $(\mathrm{mmHg})$, both systolic and diastolic, from the vertical axis. Normal values ${ }^{77}$ are indicated by the shaded bands, and the vertical lines delineate initial, daytime, evening, night-time and early morning periods. 


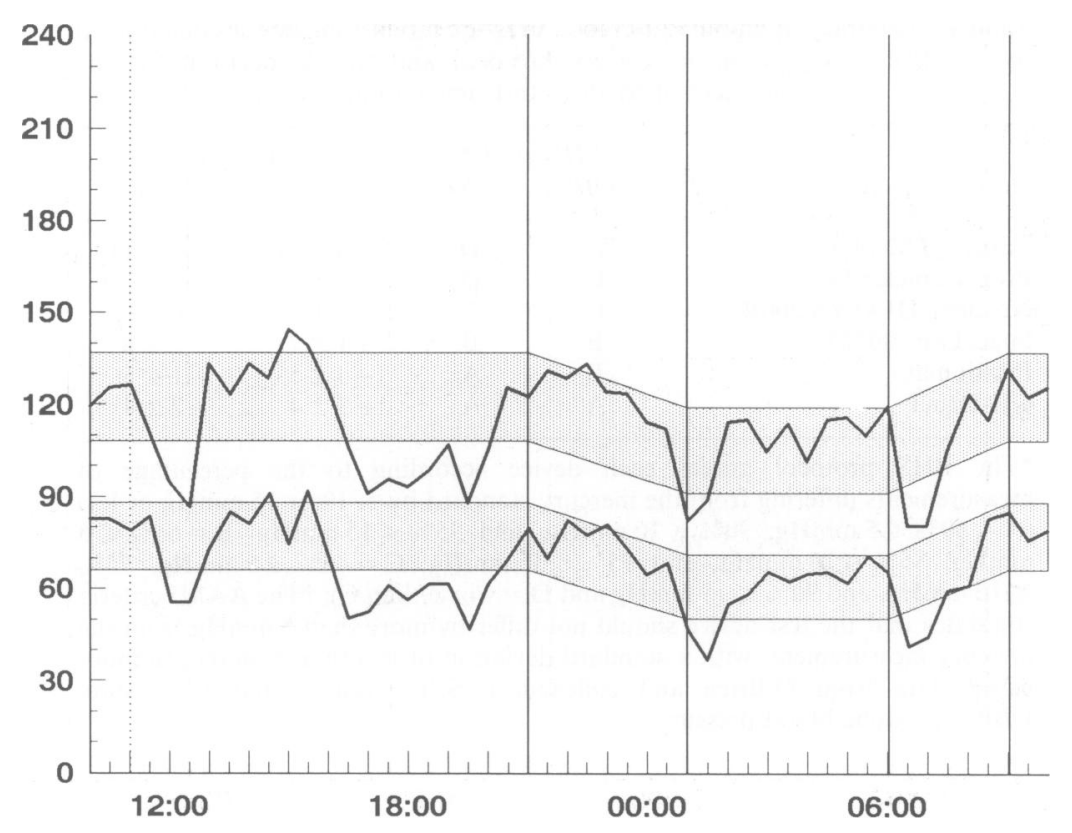

Figure 6 Twenty-four-hour ambulatory blood pressure profile from a subject with marked measurement-tomeasurement variability. Time (hours) is plotted from the horizontal axis and blood pressure ( $\mathrm{mmHg}$ ), both systolic and diastolic, from the vertical axis. Normal values ${ }^{97}$ are indicated by the shaded bands, and the vertical lines delineate initial, daytime, evening, night-time and early morning periods.

less. Prior to the main validation test the BHS protocol demands a high level of agreement between observers, assesses interdevice variability and, in addition, stipulates that the device be in use for a period of 1 month so as to ensure that device accuracy is not altered by the wear and tear of daily use. These protocols standardize independent assessments of automated devices and allow comparison of one system with another. The grading system of the BHS protocol provides a more sensitive estimate of accuracy than the AAMI standard which only allows for a pass or fail. O'Brien and colleagues comprehensively reviewed previous validation studies in $1990^{16}$ and have recently performed a number of comparative assessments, the results of which are summarized in Table I. Their data would suggest that, by both AAMI and BHS criteria, the CH-Druck device is the most accurate instrument, and that the Profilmat device and the SpaceLabs 90207 both perform satisfactorily. The other three devices, the Novocor DIASYS 200, the Pressurometer IV and the Takeda TM-2420 were less accurate.

\section{Analysis of 24-hour blood pressure data}

Noninvasive ambulatory blood pressure monitoring yields intermittent blood pressure and heart rate data, in contrast to the single values of clinical readings and the continuous data of intra-arterial pressure monitoring. Many different methods have been proposed to analyse the large amount of data provided by 24 -hour measurement. ${ }^{22,23}$ These can be classified into: (1) indices of average blood pressure such as the mean, median, or mode; (2) measure of dispersion or variability such as the range, fractiles, variance, standard deviation or the root mean squared successive differences; (3) methods of pattern analysis which include mean day-to-night differences, curve smoothing procedures or cumulative sums techniques; and (4) miscellaneous methods such as displaying the data as a frequency histogram or calculation of the load.

\section{Measures of average blood pressure}

The circadian pattern present in most 24-hour blood pressure profiles (Figures 1, 3-5) ${ }^{24-27}$ results in bimodal distributions on frequency histograms of blood pressure values taken over 24 hours. ${ }^{28}$ Thus neither parametric nor non-parametric measures of centrality, the mean and the median, appear to adequately describe average 24-hour blood pressure behaviour. The two modes of the frequency histogram can be stated, or alternatively two new models may be utilized. An updated cumulative sums technique ${ }^{29}$ allows identification 
Table I Accuracy of ambulatory blood pressure monitoring devices determined by the British Hypertension Society protocol and the Association for the Advancement of Medical Instrumentation criteria

\begin{tabular}{lcccc}
\hline & \multicolumn{2}{c}{$B H S$ grade* } & \multicolumn{2}{c}{$A A M I^{\dagger}$} \\
Device & $S B P$ & $D B P$ & $S B P$ & $D B P$ \\
\hline Takeda TM-2420 & D & D & $-4 \pm 11$ & $-2 \pm 11$ \\
Pressurometer IV & C & D & $-2 \pm 11$ & $-3 \pm 11$ \\
Novacor DIASYS 200R & C & C & $-1 \pm 8$ & $0 \pm 8$ \\
SpaceLabs 90207 & B & B & $-1 \pm 7$ & $-3 \pm 6$ \\
Profilomat & B & A & $-3 \pm 5$ & $-1 \pm 5$ \\
CH-Druck & A & A & $-3 \pm 4$ & $-2 \pm 4$ \\
\hline
\end{tabular}

*The BHS protocol grades each device according to the percentage of measurements differing from the mercury standard by 5,10 or $15 \mathrm{mmHg}$ or less $(\mathrm{A}=80 \% \leqslant 5 \mathrm{mmHg}, 90 \% \leqslant 10 \mathrm{mmHg}$, and $95 \% \leqslant 15 \mathrm{mmHg}: \mathrm{B}=65 \% \leqslant 5$ $\mathrm{mmHg}, 85 \% \leqslant 10 \mathrm{mmHg}$, and $95 \% \leqslant 15 \mathrm{mmHg} ; \mathrm{C}=45 \% \leqslant 5 \mathrm{mmHg}, 75 \%$ $\leqslant 10 \mathrm{mmHg}$, and $90 \% \leqslant 15 \mathrm{mmHg}$ and $\mathrm{D}=$ worse than $\mathrm{C}$ ); $†$ The AAMI criteria stipulates that the test device should not differ by more than $5 \mathrm{mmHg}$ from the mercury measurement, with a standard deviation of less than $8 \mathrm{mmHg}$ (unpublished data from O'Brien and colleagues); SBP = systolic blood pressure; $\mathrm{DBP}=$ diastolic blood pressure.

of crest and trough blood pressures, the 6-hour periods of highest and lowest average pressures, respectively, while the square wave fit method $^{30}$ describes the 24-hour blood pressure profile as a square wave with a single period of high pressure and a single period of low pressure.

\section{Measures of dispersion}

Both short-term pressure variability and circadian patterns, in addition to average blood pressure levels, may influence independently morbidity and mortality in hypertension. Conventional measures of variability, the standard deviation and the interquartile range are influenced substantially by both circadian trends and by shorter term variability. By contrast the root of the mean squared successive differences is an almost pure measure of short-term blood pressure variability. ${ }^{31}$ It is very resistant to slow rhythms and linear trends. It provides a very sensitive index of measurement-to-measurement variability, from intermittent blood pressure data.

\section{Pattern analysis}

Discussions still abound as to the optimal quantification of circadian trends in 24-hour blood pressure data. ${ }^{32}$ The difference between mean daytime and night-time pressures is a commonly used measure, but circadian variation is not well modelled by clock time and interpatient variation in the timing of periods of activity and sleep introduces errors. Analyses of the circadian pattern according to the oscillator model, such as the cosinor method, ${ }^{33,34}$ Fourier analysis, ${ }^{35}$ or periodic spline functions, ${ }^{36}$ assume incorrectly an endogenous rhythm. ${ }^{37}$ These methods are exceedingly complex and in reality are nothing more than statistical techniques describing the level of bloo pressure as a function of time throughout the daye Cumulative sums techniques, ${ }^{29}$ and square wav fitting ${ }^{30}$ neither of which are constrained by clock time, may be utilized to calculate circadian change as the difference between high and low pressure periods.

Coats and colleagues summarized the situation well:23 'Many statistical techniques can be used to describe different aspects of ambulatory records, but it is highly unlikely that any single parameter will suffice. A more realistic aim is for a combination of measures that combine statistical validity with physiological meaning and clinical usefulness This will require compromises between conflicting demands, and only experience and research will ultimately determine the best summary statistics.

\section{Clinic blood pressures, ambulatory blood pressure values and prognosis}

Clinic blood pressure measurements have long been the mainstay of hypertension diagnosis and management, owing to the weight of epidemiological evidence linking elevated clinic blood pressure readings with cardiovascular morbidity and mortality. ${ }^{38-41}$ However, although the level of arterial pressure as measured at the clinic is an important risk factor in populations, its predictive value in individual patients is poor. ${ }^{38-41}$ This poor 
predictive power of clinic pressure readings may be due to the multifactorial nature of the pathogenesis of cardiovascular damage. Alternatively or additionally, any lack of accuracy of clinic pressure measurements in reflecting an individual's true average blood pressure would greatly weaken any association between blood pressure level and risk. Clinic pressure readings may not precisely reflect true average pressures for two principal reasons: firstly, the marked moment-to-moment variability of blood pressure; $;^{42-44}$ and secondly a phenomena known as the white coat effect, whereby a subject responds to the circumstance of blood pressure measurement. ${ }^{45-47}$

The white coat phenomenon has been most forcefully demonstrated in two studies by Mancia and colleagues. ${ }^{45,46}$ They showed that when a physician prepared to take a blood pressure reading with a mercury sphygmomanometer on one arm of the patient, there was a marked rise in intra-arterial pressure in the other arm, which persisted throughout the measurement procedure. The average rise in pressure evoked by the presence of a physician was $23 / 18 \mathrm{mmHg}$. White coat hypertension has been found to be present in as many as $25 \%$ of patients who carry a 'diagnosis' of hypertension. ${ }^{47,48}$

Only the first few readings of 24-hour ambulatory blood pressure monitoring appear subject to this alerting response (Figure 5) and averaging of multiple blood pressure readings both reduces the influence of these first few readings and reduces variability, thereby improving the estimate of true average blood pressure. ${ }^{49,50}$ Hence, it would not be surprising if ambulatory blood pressure readings, averaged over 24-hours, over awake or over sleep periods, were better predictors of cardiovascular risk than clinical readings. This important question has only been addressed by the study of Perloff and colleagues, ${ }^{51}$ who showed that patients with elevated clinic blood pressure readings but normal daytime values had a similar prognosis to subjects with normal clinical and normal daytime readings, while those with elevated clinical and elevated daytime ambulatory values were at greater risk of cardiovascular events and death. Two important multicentre European studies are currently underway, the 'Office versus Ambulatory Trial' (OvA) $)^{52}$ and the 'Systolic Hypertension in the Elderly Study' (SYST-EUR), ${ }^{52,53}$ and both include in their aims the assessment of the correlation between treated 24-hour blood pressure and longterm prognosis.

In support of Perloff's unique study there are now many studies showing stronger correlations between hypertensive target organ damage and ambulatory pressures than between organ damage and clinic readings. ${ }^{54-63}$ As it is already well established that target organ damage in hypertension predicts cardiovascular risk, ${ }^{64-69}$ the likelihood is that the study of Perloff et al., showing ambulatory blood pressure to be a better predictor for stroke, heart attack and death than clinical measurement, will soon be confirmed (Figure 7).

It has been suggested that various patterns of blood pressure behaviour (Figures 1-6) might independently influence cardiovascular risk. Parati and colleagues $^{70}$ have provided convincing evidence, from inter-arterial pressure studies, that short-term blood pressure variability is positively correlated with end organ damage independently of the average 24-hour pressure level - those individuals with an ambulatory blood pressure profile such as that in Figure 6 would be at greater risk of organ damage and cardiovascular complications than those with a profile as in Figure 1.

Blood pressure usually follows a circadian rhythm (Figures 1, 3-5), ${ }^{24-27}$ with pressure levels higher during the day and lower at night. The usual nocturnal fall in blood pressure is diminished or absent in some subjects, and this has led to adoption of the terms 'dippers' (for the normal pattern) and 'non-dippers'. A blunted circadian pattern (Figure 2) has been reported to be associated with increased prevalence of left ventricular hypertrophy, ${ }^{71-74}$ atherosclerosis ${ }^{71}$ and stroke, ${ }^{75,76}$ but matters are confounded by the possibility that those with accentuated circadian blood pressure changes (Figure 4) might also be at increased risk. Blood pressure peaks may influence the development of hypertensive target organ damage, ${ }^{77,78}$ and may also trigger such events as the rupture of an

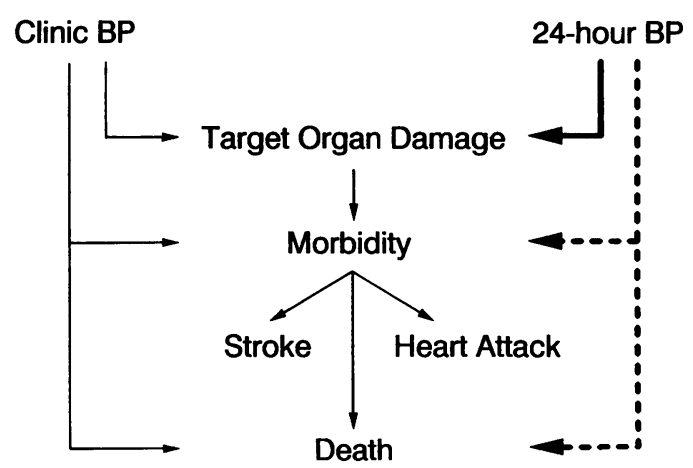

Figure 7 Scheme of reasoning the relationship of clinical blood pressure and ambulatory blood pressure to target organ damage, morbidity and mortality. The left-hand arrows indicate the established relationship between clinical blood pressure and target organ damage, and morbidity and mortality: the right-hand arrows indicate the established relationship of 24-hour ambulatory blood pressure with target organ damage and the likely, but as yet fully proven, relationship with morbidity and mortality. 
atheromatous plaque..$^{79-84}$ An excessive nocturnal reduction in blood pressure may critically reduce perfusion of vital organs, predisposing to cardiac, ${ }^{85-91}$ limb $^{92}$ and cerebral ischaemia..$^{93,94}$

Hence, while it is very probable that mean 24-hour ambulatory blood pressure levels will prove to be a better predictor of cardiovascular risk than clinical readings, the issue of the influence of spontaneous variability, circadian patterns, and night-time versus daytime blood pressure readings on morbidity and mortality is far from clear.

\section{Twenty-four-hour blood pressure monitoring in clinical practice}

Ambulatory blood pressure measurement is currently evolving from being purely a research tool into clinical practice. In order to avoid misuse, it is essential that all operators have an understanding of the normal ranges of ambulatory blood pressure readings, ${ }^{95-98}$ as well as the typical moment-tomoment blood pressure variabilities ${ }^{42-44,99}$ and usual circadian rhythms. ${ }^{24-27}$ In addition, the operator must be familiar with the equipment and with the calibration procedures. $\mathrm{He} / \mathrm{she}$ must be prepared to give the necessary time to instruct the subject so that as many measurements as possible are obtained during the recording period. Subjects for ambulatory blood pressure measurement must be capable of coping with and caring for the recorder. The conditions of measurement for the subject should be standardized as far as possible in relation to activity; in particular the arm should be held still during each measurement; ${ }^{100}$ a similar level of activity should be undertaken for comparative repeat measurements; working days should not be compared with recreational days and likewise in shift workers, comparative measurements should be made between similar shifts. ${ }^{32,33}$ The subjects should be asked to keep a diary of activities during the recording period, unless motion-logging, as an objective assessment of activity, is available. ${ }^{101}$

The clinical indications for using 24-hour noninvasive blood pressure measurement are best considered in relation to the diagnosis of hypertension or hypotension, and the selection and evaluation of anti-hypertensive drug treatment.

\section{Diagnosis of hypertension}

Normal values over 24 hours for adults according to gender and age have now been defined. ${ }^{95-98}$ Values for women during pregnancy are soon to be published. ${ }^{102}$ Hence by comparison with this normative data, monitoring is particularly helpful in deciding whether subjects with a borderline elevation in clinical blood pressure should be labelled normotensive or hypertensive. ${ }^{103}$ These subjects are currently likely to receive possibly unnecessary treatment on the basis of clinical readings, and may be penalized for insurance cover and employment. ${ }^{48}$ As mentioned above ambulatory monitoring is an effective non-invasive method of determining whether blood pressure elevation is due solely to a white-coat effect. ${ }^{47}$ If it is accepted that white-coat hypertensives do not need treatment, at least in the early stages, there is a prospect that 24-hour monitoring of blood pressure could be very cost-effective - the additional costs of the assessment would be more than offset by reductions in the numbers of patients perceived as needing anti-hypertensive drug treatment. ${ }^{104}$ Elderly subjects with isolated systolic hypertension diagnosed by conventional methods are frequently found to have much reduced or essentially normotensive systolic pressures by ambulatory blood pressure monitoring. ${ }^{105}$ The Syst-Eur study ${ }^{53}$ results, when available, should clarify if these patients with elevated clinical systolic readings but normal ambulatory systolic values are at increased cardiovascular risk, and whether they should receive any anti-hypertensive medication. Patients with secondary hypertension frequently exhibit a non-dipping ambulatory blood pressure profile as in Figure 2 but as many patients with essentiab hypertension also have blunted circadian rhythms ambulatory monitoring can not be used as specific screening test for secondary hyperten? sion. ${ }^{106,107}$

Although not proven as yet, hypertensive patients with both attenuated and excessive circadian swings in blood pressure may be at in creased risk of cardiovascular complications. ${ }^{72-94,108}$ The only way of determining dipper status is by performing 24-hour blood pressure monitoring. ${ }^{108}$

\section{Diagnosis of hypotension}

Orthostatic hypotension, characterized by standing hypotension in conjunction with supine normotension or even hypertension, is not always easily detected in the clinical or office setting, and ambulatory blood pressure monitoring may play a valuable role in the diagnosis and assessment of therapeutic effects in patients with this condition. ${ }^{109}$

\section{Selection and evaluation of anti-hypertensive drug treatment}

Ambulatory blood pressure monitoring provides an assessment of anti-hypertensive drug effect over 24 hours. ${ }^{110}$ There is some evidence that different groups of drugs may have different effects on the 24-hour blood pressure profile. ${ }^{111}$ Beta adrenergic blocking agents may blunt the circadian pattern and angiotensin converting enzyme inhibitors may 
accentuate the nocturnal dip in blood pressure. ${ }^{112}$ and thus these two drug groups may be most suited to treating patients with marked daytime hypertension and those with a sustained elevation of pressure over the entire 24 hours, respectively. Evaluation of the 24-hour profile is particularly helpful in the assessment of drug efficacy in individuals in whom clinical readings consistently suggest poor control, despite intensive drug therapy (resistant hypertensives), and in patients with hypotensive symptoms, so as to guard against excessive therapy. ${ }^{103,113}$ Many patients whose hypertension was initially diagnosed by office measurement and whose blood pressure has been well controlled may well merit a drug-free period which can be assessed with safety by periodic 24 -hour pressure monitoring.

A major issue to be resolved prior to 24-hour blood pressure monitoring realizing its full clinical potential in the evaluation of patients, is the recognition of the procedure by reimbursing agencies. While most experts in the field believe that 24-hour monitoring does have an important clinical role, there is also concern that the technique might be used excessively, particularly if the physician has a direct financial incentive to perform the test. As the number of patients with questionable blood pressure elevations is so enormous, and with ever-increasing interest and pressure from public and private health-care providers and from the pharmaceutical industry, it is important to lay down guidelines for the application of ambulatory blood pressure monitoring in clinical practice. ${ }^{103,114,115}$

\section{Twenty-four-hour blood pressure monitoring and clinical drug trials}

Ambulatory blood pressure monitoring can improve and simplify clinical trials of anti-hypertensive drugs in a number of ways. Firstly, ambulatory monitoring allows identification and exclusion of those patients in whom the blood pressure is raised only in the clinic environment. ${ }^{45-48}$ These so-called 'white-coat hypertensives' do not have sustained hypertension and appear to respond differently to anti-hypertensive drugs and develop more side effects. ${ }^{116}$

Secondly, an important difference between conventional and ambulatory blood pressure measurement is the absence of a placebo response with the latter. ${ }^{117,118}$ The placebo response, whereby the administration of placebo is associated with a reduction in blood pressure, may be an artifact of clinical blood pressure measurement due to the inherent variability of arterial pressure resulting in regression to the mean or may be due to increasing familiarity of the patient with the clinical environ- ment. In uncontrolled drug studies where clinical blood pressures are used as the measure of response, the reduction in blood pressure due to a placebo response may be attributed erroneously to the drug. The absence of placebo response with noninvasive ambulatory measurement should facilitate simplification of the design of efficacy studies of anti-hypertensive drugs, in that long placebo phases may be omitted.

Thirdly, averaging the multiple blood pressure readings so as to obtain a mean 24-hour ambulatory blood pressure value reduces variability as compared to single clinic readings, thereby improving the repeatability of blood pressure estimations. ${ }^{49}$ In a study by Conway and Coats on 75 subjects the standard deviation of the difference in diastolic pressure between two clinic readings taken a month apart was $12.3 \mathrm{mmHg}{ }^{50} \mathrm{On} 24$-hour monitoring this difference fell to $6.3 \mathrm{mmHg}$. Since the number of subjects needed in a trial is related to the square of the standard deviation of the difference, the improved repeatability leads to a substantial reduction in the number of subjects needed.

Lastly, and perhaps most importantly, ambulatory blood pressure monitoring can provide a comprehensive assessment of the pattern of antihypertensive drug effect over time, ${ }^{119,120}$ clarifying their effects on circadian pattern, ${ }^{111}$ and revealing any excessive lowering of blood pressure. ${ }^{113}$

In view of these many advantages of ambulatory blood pressure monitoring over clinical readings in the arena of clinical drug trials, it is somewhat surprising that the governmental regulating agencies in the United States and Europe have not approved, nor published guidelines for the use of ambulatory blood pressure monitoring in the evaluation of anti-hypertensive treatment, and have continued to seek casual blood pressure data alone. While the United States Food and Drugs Administration does not appear likely to approve ambulatory monitoring in the near future, as it finds that 'No drug development data have been submitted where ambulatory blood pressure data have been found useful in decision-making, ${ }^{121}$ proposals for European Commission guidelines that might make ambulatory pressure data a mandatory component of new drug applications, are being considered. ${ }^{122}$

\section{Conclusions}

Noninvasive blood pressure monitoring has so many advantages over other available methods of blood pressure evaluation in the clinical diagnosis and management of individual hypertensive patients, and in the evaluation of anti-hypertensive drug efficacy that the technique is now becoming 
established in clinical practice. The most important question now awaiting an answer is which of the many aspects of blood pressure behaviour that are now quantifiable by noninvasive 24-hour ambulatory monitoring, will prove best in predicting long-term morbidity and mortality.

\section{References}

1. Hinman, A.T., Engel, B.T. \& Bickford, A.F. Portable blood pressure recorder. Accuracy and preliminary use in evaluating intradaily variations in pressure. Am Heart $J$ 1962, 63: 663-668.

2. Hales, S. Statistical Essays: Containing Haemosticks, Vol. ii. W. Innys and R. Manby, London, 1933, p.l et seq.

3. Poiseuille, J.L.M. Recherches sur la force du couer aortique. J Physiol Exp 1828, 8: 272.

4. Bevin, A.T., Honour, A.J. \& Stott, F.H. Direct arteria pressure recording in unrestricted man. Clin Sci 1969, 36: 329-334.

5. Littler, W.A., Honour, A.J., Sleight, P. \& Stott, F.D. Continuous recording of direct arterial pressure and electrocardiogram in unrestricted man. $\mathrm{Br}$ Med J 1972, 3: 76-78.

6. O'Brien, E. \& Fitzgerald, D. The history of indirect blood pressure measurement. In: O'Brien, E. \& O'Malley, K. (eds) Handbook of Hypertension, Vol. 14, Blood Pressure Measurement. Elsevier Science Publishers, Amsterdam, 1991, BV $1-54$.

7. Riva-Rocci, S. Un nuovo sfigmomanometro. Gazz Med Torino 1896, 50: 981-996; 51: 1000-1017.

8. Korotkov, N.C. To the question of methods of determining the blood pressure (from the clinic of Professor C.P. Federoff). Rep Imp Mil Acad 1905, 11: 365-367.

9. Janeway, T.C. The Clinical Study of Blood Pressure. D. Appleton \& Co, New York, London, 1904, pp. 84-87.

10. Morgan, J.L., Kemmerer, W.T. \& Halbin, M.D. Doppler shifted ultrasound. History and application in clinical medicine. Minn Med 1969, 52: 503.

11. Hunt, J.C., Frohlich, E.D., Moser, M., Roccella, E.J. \& Keighley, E.A. Devices used for self-measurement of blood pressure: revised statement of the National High Blood Pressure Education Program. Arch Intern Med 1985, 145: 2231-2234.

12. Kaplan, N.M. Misdiagnosis of systemic hypertenion and recommendations for improvement. Am J Cardiol 1987, 60: $1383-1386$

13. Canadian coalition for high blood pressure prevention and control: recommendations on self-measurement of blood pressure. Can Med Assoc J 1988, 138: 1093-1096.

14. World Hypertension League. Self measurement of blood pressure. Clin Exp Hypertens (A) 1989, 11: 167-171.

15. Kain, H.K., Hinman, A.T. \& Sokolow, M. Arterial blood pressure measurements with a portable recorder in hypertensive patients. Variability and correlation with casual pressures. Circulation 1964, 30: 882-892.

16. O'Brien, E. \& O'Malley, K. Twenty-four-hour ambulatory blood pressure monitoring: A review of validation data. $J$ Hypertens 1990, 8 (Suppl 6): S11-S16.

17. Yamokoshi, K., Rolfe, P. \& Murphy, C. Current developments in non-invasive measurements of arterial blood pressure. J Biomed Eng 1988, 10: 130-137.

18. Di Rienzo, M., Grassi, G., Pedotti, A. \& Mancia, G. Continuous versus intermittent blood pressure measurements in estimating 24-hour average blood pressure. Hypertension 1983, 5: 264-269.

19. Wesseling, K.H. Finapres, continuous non-invasive finger arterial pressure based on the method of Penaz. In: MeyerSabellek, W., Analle, M., Gotzen, R. \& Steinfeld, L. (eds) Blood Pressure Measurements: New Techniques in Automatic and 24-hour Indirect Monitoring. Springer-Verlag, New York, 1990, pp.161-172.

20. Association for the Advancement of Medical Instrumentation. American National Standard for Electronic or Automated Sphygmomanometers. AAMI, Washington, DC, 1987.

21. O'Brien, E., Petrie, J., Littler, W. et al. The British Hypertension Society protocol for the evaluation of automated and semi-automated blood pressure measuring devices with special reference to ambulatory systems. J Hypertens 1990,8 607-619.

22. White, W.B. Analysis of ambulatory blood pressure data in antihypertensive drug trials. J Hypertens 1991, 9 (Suppl 1): S27-S32.

23. Coats, A.J.S., Clark, S.J. \& Conway, J. Analysis of ambulatory blood pressure data. J Hypertens 1991; 9 (Suppl 8): S19-S21.

24. Goldberg, A.D., Raftery, E.B., Cashman, P.M.M. \& Scott, F.D. Study of untreated hypertensive subjects by means of continuous intra-arterial blood pressure recordings. $B$ Heart J 1978, 40: 656-659.

25. Drayer, J.I.M., Weber, M.A., DeYoung, J.L. \& Wyle, F Circadian blood pressure patterns in ambulatory hypertensive patients. Am J Med 1982, 73: 493-499.

26. Littler, W.A., Honour, A.J., Carter, R.D. \& Sleight, P. Sleep and blood pressure. Br Med J 1975, 3: 346-348.

27. Pickering, T.G., Harshfield, G.A., Kleinert, H.D., Blank, S \& Laragh, J.H. Blood pressure during normal daily activities, sleep and exercise. Comparison of values ino normal and hypertensive subjects. JAMA 1982, 24t 992-996.

28. Littler, W.A., West, M.J., Honour, A.J. \& Sleight, P. The variability of arterial pressure. Am Heart $J$ 1978, 95: 180-186.

29. Stanton, A., Cox, J., Atkins, N., O'Malley, K. \& O'Brien, E Cumulative sums in quantifying circadian blood pressure patterns. Hypertens 1992, 19: 93-101.

30. Idema, R.N., Gelsema, E.S., Wenting, G.J., Grashuis, J.L. van den Meiracker, A.H., Brouwer, R.M.L. \& Man in 't Veld, A.J. A new model for diurnal blood pressure profiling, square wave fit compared with conventional methods. Hypertens 1992, 19: 595-605.

31. Schachinger, N., Langewitz, W., Schmieder, Re. \& Ruddel, H. Comparison of parameters for assessing blood pressure and heart rate variability from non-invasive twenty-fourhour blood pressure monitoring. J Hypertension 1989, 7: (Suppl 3): S81-S84.

32. Pickering, T.G., Schnall, P.L., Schnall, P.L., Schwartz, J.E \& Pieper, C.F. Can behavioral factors produce a sustained elevation of blood pressure? Some observations and a hypothesis. J Hypertens 1991, 9 (Suppl 8): S66-S68.

33. Halberg, F. Chronobiology. Ann Rev Physiol 1969, 31 675-725.

34. Smólensky, M., Tatan, S.E., Bergman, S. et al. Circadian rhythmic aspects of human cardiovascular function: A review by chronobiological statistical methods. Chronobiologia 1975, 3: 337-371.

35. Germano, G., Damianti, S., Ciavarella, M. et al. Detection of a diurnal rhythm in arterial blood pressure in the evaluation of 24-hour antihypertensive therapy. Clin Cardiol 1984, 7: 525-535. 
36. Streitberg, B., Meyer-Sabellek, W. \& Baumgart, P. Statistical analysis of circadian blood pressure recordings in controlled clinical trials. J Hypertens 1989, 7: (Suppl 3): S11-S17.

37. Baumgart, P. Twenty-four-hour blood pressure is not dependent on endogenous circadian rhythm. J Hypertens 1989, 7: 331-334.

38. Stamier, J., Rhomberg, P., Schoenberger, J.A. et al. Multivariate analysis of the relationship of seven variables to blood pressure. Findings of the Chicago Heart Assn Detection Project in Industry, 1967-1972. J Chron Dis 1975, 28: 527-548.

39. Reid, D.D., Hamilton, P.J.S., McCartney, P. \& Rose, G. Smoking and other risk factors for coronary heart disease in British civil servants. Lancet 1976, 2: 979-984.

40. Kagan, A., Harris, B.R. \& Winkelstein, W. Epidemiologic studies of coronary disease and stroke in Japanese men living in Japan, Hawaii and California: Demographic, physical, dietary and biochemical characteristics. J Chron Dis 1974, 27: 345-364.

41. Dawber, T.R. The Framingham Study. The Epidiemiology of Atheroslerotic Disease. Harvard University Press, Cambridge, 1980.

42. Richardson, D.W., Honour, A.J., Fenton, G.W., Stott, F.H. \& Pickering, G.W. Variation in arterial pressure throughout the day and night. Clin Sci 1964, 26: 445-460.

43. Schmieder, R.E., Lavie, C.J. \& Messerli, F.H. The variability of arterial pressure; What is the clinical importance? Cardiovasc Rev Rep 1987, 8: 65-69.

44. Pickering, T.G. Strategies for the evaluation and treatment of hypertension and some indications of blood pressure variability. Circulation 1987, 76 (Suppl 1): 177-182.

45. Mancia, G., Grassi, G., Pomidossi, G. et al. Effects of blood pressure measurement by the doctor on patient's blood pressure and heart rate. Lancet 1983, 2: 695-697.

46. Mancia, G., Parati, G., Pomidossi, G., Grassi, G., Casadei, R. \& Zanchetti, A. Alerting reaction and rise in blood pressure during measurement by physician and nurse. Hypertension 1987, 9: 209-215.

47. Pickering, T.G., James, G.D. Boddie, C., Harshfield, G.A., Blank, S. \& Laragh, J.H. How common is white coat hypertension? JAMA 1988, 259: 225-228.

48. O'Brien, E. \& O'Malley, K. Overdiagnosing hypertension. Br Med J 1988, 297: 1211.

49. Conway, J., Johnson, J., Coats, A., Somers, V. \& Sleight, P. The use of ambulatory blood pressure monitoring to improve accuracy and reduce the numbers of subjects in clinical trials of antihypertensive agents. J Hypertens 1988, 6: $111-116$.

50. Conway, J. \& Coats, A. Value of ambulatory blood pressure monitoring in clinical pharmacology. J Hypertens 1989, 7 (Suppl 3): S29-S32.

51. Perloff, D., Sokolow, M. \& Cowan, R. The prognostic value of ambulatory blood pressures. JAMA 1983, 249: 2792-2798.

52. Clement, D.L., De Buyzere, M. \& Duprez, D.D. Ambulatory blood pressure and prognosis: summary of ongoing studies. J Hypertens 1991, 9 (Suppl 8): S51-S53.

53. Staessen, J., Amery, A. \& Birgenhager, W. SYST-EUR. A multicentre trial on the treatment of isolated systolic hypertension in the elderly: first interim report. J Cardiovasc Pharmacol 1992, 19: 120-125.

54. Rowlands, D.B., Glover, D.R., Ireland, M.A. et al. Assessment of left ventricular mass and its response to antihypertensive treatment. Lancet 1982, i: 467-470.

55. Lattuada, S. Rindi, M., Antivalle, M., Mutinelli, M.R., Corallo, S. \& Libretti, A. Ambulatory blood pressure monitoring (24h), basal blood pressure and left ventricular echocardiographic findings in young adults. $J$ Hypertens 1985, 3 (Suppl 3): S339-S341.
56. De Gaudemaras, R., Camaleonta, A., Dimitriou, R., Debru, J.L. \& Mallion, J. Interest of ambulatory blood pressure, exercise test recordings and echocardiographic measurements, in borderline arterial hypertension. In: Germano, O. \& Edizone, L. (eds) Blood Pressure Recording in the Clinical Management of Hypertension, Pozzi, Rome, 1985, pp. 46-49.

57. Gosse, P., Campobello, G., Aoinzerate, E., Roudaut, R., Broustat, J.P. \& Dallochio, M. Left ventricular hypertrophy in hypertension: correlation with rest, exercise and ambulatory systolic blood pressure. J Hypertension 1986, 4 (Suppl 5): S297-299.

58. Prisant, L.M. \& Carr, A.A. Ambulatory blood pressure monitoring and echocardiographic left ventricular wall thickness and mass. Am J Hypertens 1990, 3: 81-89.

59. Pannier, B., Brunet, P., El Aroussy, W. et al. Pulse pressure and echocardiography findings in essential hypertension. $J$ Hypertens 1989, 7: 127-132.

60. Dimmit, S.B., West, J.N.W., Eames, S.M., Gibson, J.M. Gosling, P. \& Littler, W.A. Usefulness of ophthalmoscopy in mild to moderate hypertension. Lancet 1989, i: 1103-1106.

61. Shimada, K., Kawanoto, A., Matsubayishi, K. \& Ozawa, T. Silent cerebrovascular disease in the elderly: correlation with ambulatory pressure. Hypertension 1990, 16: 692-699.

62. Opsahl, J.A., Abraham, P.A., Halstonson, C.E. \& Keane, W.F. Correlation of office and ambulatory blood pressure measurements with urinary albumin and $\mathrm{N}$-acetyl- $\mathrm{\beta}$-dglucosaminidase excretions in essential hypertension. $\mathrm{Am} \mathrm{J}$ Hypertens 1988, 1: 117S-120S.

63. Giaconi, S., Levanti, C., Fommei, E. et al. Microalbuminuria and casual and ambulatory blood monitoring in normotensives and in patients with borderline and mild essential hypertension. Am J Hypertens 1989, 2: 259-261.

64. Kernel, W.B., Gordon, T. \& Offut, D. Left ventricular hypertrophy by electrocardiogram. Prevalence, incidence and mortality in the Framingham Study. Ann Intern Med 1969, 71: 89-105.

65. Levy, D., Garrison, R.J. Savage, D.D. Kannel, W.B. \& Castelli, W.P. Prognostic implications of echocardiographically determined left ventricular mass in the Framingham Heart Study. $N$ Engl J Med 1990, 322: 1561-1566.

66. Casale, P.N., Devereux, R.B., Milner, M. et al. Value of echocardiographic measurement of left ventricular mass in predicting cardiovascular morbid events in hypertensive men. Ann Intern Med 1986, 105: 173-178.

67. Nestrova, A.L., Nosikov, I.D. \& Yurenev, A.P. Prognostic significance of blood pressure and left ventricular hypertrophy in systemic and non-systemic treatment of essential hypertension. Kardiologia 1986, 8: 89-91.

68. Koren, M.J., Devereux, R.B., Casale, P.N., Savage, D.D. \& Laragh, J.H. Relation of left ventricular mass and geometry to morbidity and mortality in men and women with essential hypertension. Ann Intern Med 1991, 114: 345-352.

69. Ljungman, $S$. Microalbuminuria in essential hypertension. Am J Hypertens 1990, 3: 956-960.

70. Parati, G., Pomidossi, G., Albini, F., Malaspina, D. \& Mancia, G. Relationship of 24-hour blood pressure mean and variability to severity of target organ damage in hypertension. J Hypertens 1987, 5: 93-98.

71. Kobrin, I., Oigman, W., Kuman, A. et al. Diurnal variation of blood pressure in elderly patients with essential hypertension. J Am Geriatr Soc 1984, 32: 896-899.

72. Verdecchia, P., Schillaci, C., Guerreri, M. et al. Circadian blood pressure changes and left ventricular hypertrophy in essential hypertension. Circulation 1990, 81: 528-536.

73. Klein, W., Zweiker, R., Eber, B., Dusleag, J., Brussee, H. \& Rotman, B. Circadian blood pressure pattern in patients with treated hypertension and left ventricular hypertrophy. Angiology 1991, 42: 379-386. 
74. Suzuki, Y., Kuwajima, I., Kanemaru, A. et al. The cardiac functional reserve in elderly hypertensive patients with abnormal diurnal change in blood pressure. $J$ Hypertens 1992, 10: 173-179.

75. O'Brien, E., Sheridon, J. \& O'Malley, K. Dippers and non-dippers (letter). Lancet 1988, 2: 397.

76. Shimada, K., Kawamoto, A., Matsubayashi, K., Nishinaga, M., Kimura, S. \& Ozawa, T. Diurnal blood pressure variations and silent cerebrovascular damage in elderly patients with hypertension. J Hypertens 1992, 10: 875-878.

77. Devereux, R.B., Pickering, T.G. \& Harshfield, G.A. Left ventricular hypertrophy in patients with hypertension. Importance of blood pressure response to regularly recurring stress. Circulation 1983, 68: 470-476.

78. Gottdiener, J.S. Brown, J., Zoltick, J. \& Fletcher, R.D. Left ventricular hypertrophy in men with normal blood pressure: relation to exaggerated blood pressure response to exercise. Ann Intern Med 1990, 112: 161-166.

79. Friedman, M. The pathogenesis of coronary plaques, thrombosis, and haemorrhages: an evaluative review. Circulation 1975, 52: (Suppl 3): 34-40.

80. Miller-Criag, M.W., Bishop, C.N. \& Raftery, E.B. Circadian variation of blood pressure. Lancet 1978, i: 795-797.

81. Myers, A. \& Dewar, H.A. Circumstances attending 100 sudden deaths from coronary artery disease with coroner's necropsies. Br Heart J 1975, 37: 1133-1143.

82. Pedoe, H.T., Clayton, D., Morris S.N. et al. Coronary heart attacks in East London. Lancet 1975, 2: 833-838.

83. Muller, J.E., Tofler, G.H. \& Stone, D.H. Circadian variation and triggers of onset of acute cardiovascular disease. Circulation 1989, 79: 733-743.

84. Agnoli, A., Manfredi, M., Mossuto, L. et al. Rapport entre les rythmes hemeronyctaux de la tension arterielle, et sa pathogenie de l'insuffisance vasculaire cerebrale. Rev Neurol 1975, 131: 597-606.

85. Floras, J.S. Antihypertensive treatment, myocardial infarction and nocturnal myocardial ischaemia. Lancet 1988, 2: 944-946.

86. Harrison, D.G., Florentine, M.S., Brooks, L.A., Cooper, S.M. \& Marcus, M.L. The effect of hypertension and left ventricular hypertrophy on the lower range of coronary autoregulation. Circulation 1988, 77: 1108-1115.

87. Editorial. Coronary artery disease in hypertension. Lancet 1988, ii: $1461-1462$

88. Brush, J.E., Cannon, R.O., Schenke, W.H. et al. Angina due to coronary microvascular disease in hypertensive patients without left ventricular hypertrophy. N Engl J Med 1988, 319: $1302-1307$.

89. Opherk, D., Mall, G., Zebe, H. et al. Reduction of coronary reserve: a mechanism for angina pectoris in patients with arterial hypertension and normal coronary arteries. Circulation 1984, 69: 1-7.

90. Pepi, M., Alimento, M., Maltagliati, A. \& Guazzi, M.D. Cardiac hypertrophy in hypertension: repolarization abnormalities elicited by rapid lowering of pressure. Hypertens 1988, 11: 84-91.

91. Yagil, Y., Kobrin, I., Lebsel, B. \& Ben-Ishay, D. Ischaemic ECG changes with initial nifedipine therapy of severe hypertension. Am Heart J 1982, 103: 310-311.

92. Jelnes, R., Bulow, J., Tinnesen, K.H., Lassen, N.A. \& Holstein, P. Why do patients with severe arterial insufficiency get pain during sleep? Scand J Clin Lab Invest 1987, 47: 649-654.

93. Standgaard, S. Autoregulation of cerebral blood flow in hypertensive patients: the modifying influence of prolonged antihypertensive treatment on the tolerance to acute druginduced hypotension. Circulation 1976, 53: 720-727.

94. Marshall, J. Diurnal variation in occurrence of strokes. Stroke 1977, 8: 230-231.

95. Palatini, P. \& Pessina, A. A new approach to define the upper normal limits of ambulatory blood pressue. $J$ Hypertens 1990, 8 (Suppl 6): S65-S70.
96. Baumgart, P., Walger, P., Jurgens, U. \& Rahn, K.H. Reference data for ambulatory blood pressure monitoring: what results are equivalent to the established limits of office blood pressure? Klin Wochenschr 1990, 68: 723-727.

97. O'Brien, E., Murphy, J., Tyndall, A. et al. Twenty-four hour ambulatory blood pressure in men and women aged 17 to 80 years: the Allied Irish Bank Study. J Hypertens 1991, 9: $355-360$.

98. Stassen, J., Fagard, R., Lijnen, P., Thijis, L., Van Hoof, R. \& Amery, A. Reference values for ambulatory blood pressure: a meta-analysis. J Hypertens 1990, 8 (Suppl 6): S57-S64.

99. Mancia, G. \& Zanchetti, A. Blood pressure variability. In: Zanchetti, A. \& Tarazi, R.C. (eds) Pathophysiology of Hypertension: Cardiovascular Aspects. Elsevier Science Publishers, Amsterdam, 1986.

100. White, W.B., Lund-Johansen, P. \& Omvik, P. Assessment of four ambulatory blood pressure monitors and measurements by clinicians versus intraarterial blood pressure at rest and during exercise. Am J Cardiol 1990, 65: 60-66.

101. Van Egeren, L.F. Monitoring activity and blood pressure. $J$ Hypertens 1991, 9 (Suppl 8): S25-S27.

102. Halligan, A., O'Brien, E., Mee, F. et al. Normal 24 hour ABP in pregnancy. Br J Obstet Gynaecol. (In press).

103. O'Brien, E., Cox, J. \& O'Malley, K. The role of twenty-four hour ambulatory blood pressure measurement in clinical practice. J Hypertens 1991, 9 (Suppl 8): S63-S65.

104. Krakoff, L.R., Eison, H., Phillips, R.H., Leimans, S. \& Lev, S. Effect of ambulatory blood pressure monitoring on the diagnosis and cost of treatment for mild hypertension. $\mathrm{Am}$ Heart $J$ 1988, 116: 1152-1154.

105. Cox, J., O'Brien, E. \& O'Malley, K. Ambulatory blood pressure measurement in the elderly. J Hypertens 1991, 9 (Suppl 3): S73-S77.

106. Imai, Y., Abe, K., Munakata, M. et al. Does ambulatory blood pressure monitoring improve the diagnosis of secon-o $\omega$ dary hypertension? J Hypertens 1990, 8 (Suppl 6): S71-S75.

107. Padfield, P.L. \& Stewart, M.J. Ambulatory blood pressure monitoring in secondary hypertension. J Hypertens 1991, 9? (Suppl 8): S69-S71.

108. Pickering, T.G. The clinical significance of diurnal blood pressure variations, Dippers and nondippers. Circulation 1990, 81: $700-701$.

109. Schirger, A. Ambulatory blood pressure monitoring in patients with autonomic failure (Abstract). Clin Pharmacol Ther 1989, 45: 153.

110. O'Brien, E., O'Malley, K., Cox, J. \& Stanton, A. Ambulatory blood pressure monitoring in the evaluation of drug efficacy. Am Heart J 1991, 121: 999-1006.

111. Sirgo, M.A., Mills, R.J. \& De Quattro, V. Effects of antihypertensive agents on circadian blood pressure and heart rate patterns. Arch Intern Med 1988, 148: 2547-2552.

112. Stanton, A., Atkins, N., O'Malley, K. \& O’Brien, E. Antihypertensive therapy and circadian blood pressure profiles: a retrospective analysis using cumulative sums. (Submitted for publication).

113. Berglund, G. Goals of antihypertensive therapy: is there a point beyond which reduction is dangerous? Am J Hypertens 1989, 2: 586.

114. Cox, J., O'Malley, K. \& O'Brien, E. Ambulatory blood pressure measurement in general practice. Br J Gen Pract 1992, 42: 402-430.

115. O'Brien, E. \& O'Malley, K. The future. In: O'Brien, E. \& O'Malley, K. (eds) Handbook of Hypertension, Vol. 14: Blood Pressure Measurement. Elsevier Science Publishers, Amsterdam, 1991, BV 380-386.

116. Waeber, B., Scherrer, U., Petrillo, A. et al. Are some hypertensives overtreated? A prospective study of ambulatory blood pressure recording. Lancet 1987, 2: 732 .

117. Dupont, A.G., van der Niepen, P. \& Six, R.O. Placebo does not lower ambulatory blood pressure. Br J Clin Pharmacol 1987, 24: 106. 
118. Drayer, J.I.M., Weber, M.A., De Young, J.L. \& Brewer, D.D. Long-term BP monitoring in the evaluation of antihypertensive therapy. Arch Intern Med 1983, 143: 898.

119. Weber, M.A. Documentation of the effective length of action of antihypertensive treatment. Am J Cardiol 1990, 66: $43 \mathrm{C}-48 \mathrm{C}$

120. O'Brien, E., Cox, J. \& O'Malley, K. Ambulatory blood pressure measurement in the evaluation of blood pressure lowering drugs. J Hypertens 1989, 7: 243.
121. Lipicky, R. The United States Food and Drug Administration Guidelines on ambulatory blood pressure monitoring. $J$ Hypertens 1991, 9 (Suppl 8): S59.

122. Meyer-Sabellek, W.A. Proposals for European Commission guidelines on ambulatory blood pressure monitoring. $J$ Hypertens 1991, 9 (Suppl 8): S60-S62. 